\title{
Relationship between Muscle Strength and Elbow Extension Test
}

\section{Kazumasa Nakagawa*}

Department of Physical Therapy, Takasaki University of Health and Welfare, Takasaki City, Japan

*Corresponding author: Dr. Kazumasa Nakagawa, Department of Physical Therapy, Faculty of Health Care, Takasaki University of Health and Welfare, Takasaki City, Gunma Prefecture, Japan, Tel: 81-27-352-1291; E-mail: nakagawa-ka@takasaki-u.ac.jp

Rec date: Mar 29, 2014, Acc date: July 26, 2014; Pub date: July 30, 2014

Copyright: (c) 2014 Nakagawa K. This is an open-access article distributed under the terms of the Creative Commons Attribution License, which permits unrestricted use, distribution, and reproduction in any medium, provided the original author and source are credited.

\section{Summary}

We examined what muscles were related to the Elbow Extension Test (EET). For 21 healthy individuals, the muscular force required to perform the EET, as well as the strength of the trunk rotators, elbow extensors, and external and internal rotators were measured using a hand-held dynamometer. We evaluated the relationship between the results of the EET and the strength of each muscle. The results of the EET may have reflected the strength of the elbow extensors. Validation of the timing of the contraction of these muscles may be important diagnostic factors for individuals with some disorders in shoulder or elbow joint.

Keywords: Shoulder disorder; Muscle strength evaluation; Kinetic chain; Elbow extensor muscles

\section{Introduction}

Upper extremity disorders, especially shoulder disorders are largely caused by shoulder joint diblitations, rotator cuff dysfunction, or deficits in the kinetic chain of the shoulder with the lower extremities and trunk. Reportedly, postural control works against gravitational central sway during the movement of the upper extremities [1]; therefore, it is important to evaluate patients with upper extremity disorders for not only shoulder joint function but also, scapula mobility and stability, as well as trunk muscle stability [2]. It was reported that those who have shoulder problems can't often exert their muscle forces during extending their elbow, and which is thought to be caused by the kinetic chain of the shoulder with the scapula and trunk $[3,4]$. And as one of the tests which have shown to accurately reflect such a kinetic chain, the elbow extension test is often used in Japan [3, 4]. However, the empiric reliability of the measured parameters that compose these tests is insufficient. Therefore, in the present study, the relationship between muscle strength and the results of the EET were examined.

\section{Material and Methods}

We evaluated 21 healthy individuals (men, 13; women, 8; age, 16.2 \pm 1.0 years; height, $165.6 \pm 7.7 \mathrm{~cm}$; weight, $59.2 \pm 9.9 \mathrm{~kg}$ ). All subjects were informed regarding the purpose and procedure of the study, and the subjects provided written informed consent before participation.

Using a hand-held dynamometer ( $\mu$-tas F-1, ANIMA), the muscular force used while performing the EET, and the strength of the trunk rotators, elbow extensors, and external and internal rotators were measured. The EET was performed with subjects in the sitting position with both arms extended and elbows in front of the chest (shoulder, $90^{\circ}$ flexion; elbow, $90^{\circ}$ flexion; and forearms, $90^{\circ}$ supination). The elbow joints were extended against resistance provided by the examiner at the distal end of the forearm, where maximal isometric force was measured (Figure 1). The manual muscle testing method described by Daniels [3] was used to measure the strength of the elbow extensors, and external and internal rotators. The strength of the trunk rotators was measured with subjects in the sitting position (Figure 2). It was reported by Hara $[3,4]$ that this test showed positive sign if there were some differences between left and right sides, so in all conditions, maximal isometric force was measured thrice and the average values and differences between left and right sides were extracted for statistical analysis.

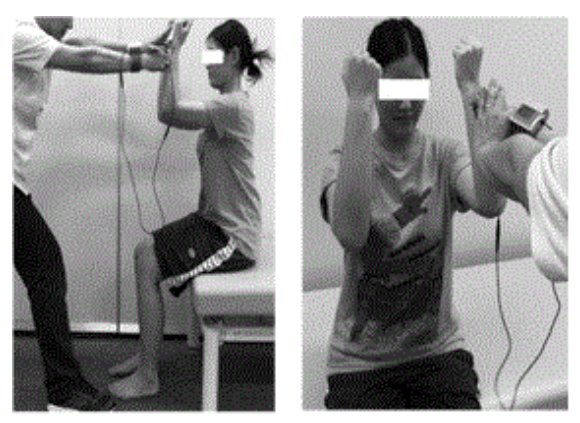

Figure 1: Elbow extension test (Lt: saggital plane, Rt: frontal plane)

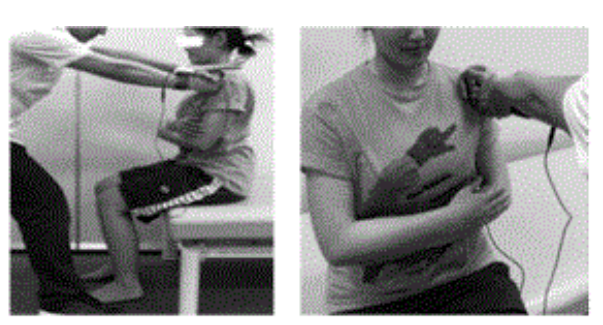

Figure 2: Measurement of the trunk rotators strength, the trunk was rotated against the resistance provided by the examiner at the acromion, where maximal isometric force was measured (Lt: saggital plane, Rt: frontal plane).

The Pearson's correlation coefficient was used in the statistical analysis to evaluate the relationship between the EET results and strength of the trunk rotators, elbow extensors, and shoulder external and internal rotators. The average values and differences between both sides were determined. All statistical analyses were performed using IBM SPSS 18.0J statistical software for Windows. A probability (p) value of $<0.05$ was considered statistically significant. 
Citation: Nakagawa K (2014) Relationship between Muscle Strength and Elbow Extension Test. Primary Health Care 4: 165. doi: 10.4172/2167-1079.1000165

Page 2 of 2

\section{Results}

Results of the muscle force in every test muscles were shown in Table 1, and as shown in Table 2, positive correlations were found between the average values of the EET and strength for each muscle and group of muscles. In the analysis of differences, positive correlations were observed between the EET results and strength of the elbow extensors.

\begin{tabular}{|l|l|l|l|l|l|}
\hline & EET & TR & EE & E/R & I/R \\
\hline average value & $147.5 \pm 30.3 \mathrm{~N}$ & $173.0 \pm 37.7 \mathrm{~N}$ & $120.4 \pm 30.1 \mathrm{~N}$ & $54.4 \pm 30.4 \mathrm{~N}$ & $113.3 \pm 39.7 \mathrm{~N}$ \\
\hline different value & $11.4 \pm 6.9 \mathrm{~N}$ & $16.8 \pm 12.1 \mathrm{~N}$ & $10.8 \pm 8.5 \mathrm{~N}$ & $5.7 \pm 5.2 \mathrm{~N}$ & $11.1 \pm 10.4 \mathrm{~N}$ \\
\hline $\begin{array}{l}\text { EET: Elbow Extension Test, TR: Trunk Rotatiors, EE: Elbow Extensors } \\
\text { E/R: External Rotators, I/R: Internal Rotators }\end{array}$ & & & \\
\hline
\end{tabular}

Table 1: The muscle force results in every test muscles

\begin{tabular}{|l|l|l|l|l|l|}
\hline \multicolumn{2}{|l|}{} & TR & EE & E/R & I/R \\
\hline \multirow{2}{*}{ EET } & average value & $\begin{array}{l}.463^{*} \\
(p=0.036)\end{array}$ & $\begin{array}{l}.785^{* *} \\
(p=0.0001)\end{array}$ & $\begin{array}{l}.739^{* *} \\
(p=0.0001)\end{array}$ & $\begin{array}{l}.576^{* *} \\
(p=0.005)\end{array}$ \\
\cline { 2 - 6 } & different value & $\begin{array}{l}-.042 \\
(p=0.858)\end{array}$ & $\begin{array}{l}.469^{*} \\
(p=0.032)\end{array}$ & $\begin{array}{l}-.305 \\
(p=0.179)\end{array}$ & $\begin{array}{l}-.048 \\
(p=0.836)\end{array}$ \\
\hline
\end{tabular}

Table 2: Results of the correlation analysis of the relationship between EET and muscle strength

\section{Discussions}

From the results of the average value, every muscle was relevant to the EET. It was easy to image that subjects who had stronger muscles could apply their muscle forces to EET because they had no problems in their shoulders. It was thought to be the reason of this significant correlation. And then, from the results of the different value, only elbow extensors were relevant to the EET. The result of the EET may have reflected the strength of the elbow extensor muscles and not reflect the coordination with another muscle in health young subjects.

As a limitation of this study, the study subjects were normal, healthy individuals without any upper extremity disorders or problems associated with the trunk muscles or other stabilizing muscles were reported to work before moving upper extremities $[1,6]$. It was reported trunk muscles have a critical role in the maintenance of stability and balance when performing movements with the extremities [7], so it is natural that not only elbow extensors, but also trunk muscles should have some kind of relation to EET. And validation of not only the muscle forces but also the timing of the contraction of these muscles may be important diagnostic factors for individuals with some upper extremity disorders.

\section{References}

1. Belen'kiÄ VE, Gurfinke' VS, Pal'tsev EI (1967) [Control elements of voluntary movements]. Biofizika 12: 135-141.

2. Kiefer A, Shirazi-Adl A, Parnianpour M (1998) Synergy of the human spine in neutral postures. Eur Spine J 7: 471-479.

3. Masafumi Hara (2005) Sports-Sensyu-no-Fuanteikata-no-Shinsatsuchou. The Journal of Sports Medicine 22: 1353-1360.

4. Masafumi Hara (2007) Toukyu-Shogaikata-ni-Taisuru-Sinsatsu-toByoutaihaku-no-Point. MB Ortop 20: 29-38.

5. Helen J Hislop (2007) Daniels and Worthingham's Muscle Testing: Techniques of Manual Examination, 8thedn. Elsevier Science Health Science Division.

6. Hodges PW, Richardson CA (1997) Feedforward contraction of transversus abdominis is not influenced by the direction of arm movement. Exp Brain Res 114: 362-370.

7. Andersson E, Swärd L, Thorstensson A (1988) Trunk muscle strength in athletes. Med Sci Sports Exerc 20: 587-593. 